\title{
A New Structural Damage Identification Method Based on Wavelet Packet Energy Entropy of Impulse Response
}

\author{
Can $\mathrm{He}^{1}$, Jianchun Xing ${ }^{1, *}$, Juelong $\mathrm{Li}^{2}$, Wei Qian ${ }^{2}$ and Xun Zhang ${ }^{1}$ \\ ${ }^{1}$ College of Defense Engineering, PLA University of Science and Technology, Nanjing, 210007, China \\ ${ }^{2}$ Technical Management Office of Naval Defense Engineering, Beijing, 100841, China
}

\begin{abstract}
Excitation makes a great influence on the wavelet energy distribution of the response signal, this deficiency leads that the traditional structural damage identification method based on wavelet energy has a low precision. In order to solve this problem, a new structural damage identification method based on wavelet packet energy entropy (WPEE) of impulse response is presented in this paper. Firstly, natural excitation technique (NExT) is adopted to extract structural impulse response. Then, WPEE of the impulse response is computed, and the change rate of WPEE is used to construct the structural damage index. An experiment of damage identification on a pile structure is provided to verify the effectiveness of the proposed method. Experiment results show that this method can accurately identify the single damage and multi-damage.
\end{abstract}

Keywords: Impulse response, structural damage identification, wavelet packet energy entropy.

\section{INTRODUCTION}

Due to strong wind force, torrential rain, severe earthquake, explosion and other abnormal loads, large-scale civil engineering may be damaged in its long service period [1]. Once structural damage occurs, it will reduce the durability of the structure, even threat the safety of the structure. Therefore, structural damage identification has become an important research topic in the engineering protection field [2]. In the past few years, many technologies have been developed to achieve structural damage identification, such as: identification methods based on time domain analysis [3, 4], identification methods based on modal parameters $[5,6]$ and identification methods based on time-frequency domain analysis [7, 8]. Among these methods, because of low entropy and multi-resolution, wavelet transform has become a popular technology in the field of structural damage identification. Kim et al. [9] found that the occurrence and development of structural damage could be accurately identified by monitoring the changes of wavelet coefficients of the response signal. Hou et al. [10] proposed a new damage identification method by detecting the singularity of the signals based on wavelet transform, and the method had been successfully applied to the Benchmark model which was provided by the American Society of Civil Engineers (ASCE). Zitto et al. [11] applied the continuous wavelet transform to analyze the acoustic emission signals from dynamic tests conducted on a reinforced concrete slab with a shaking table, the results showed this method can identify the crack in the reinforced concrete slab accurately. Ding et al. [12] firstly achieved wavelet packet energy spectrum (WPES) by decomposing

\footnotetext{
*Address correspondence to this author at the College of Defense Engineering, PLA University of Science and Technology, Nanjing, 210007, China; Tel: (86)025-80825341; Fax: (86)025-80825341; E-mail: xjc893@163.com
}

the wavelet packet of the response signal, and then computed the change rate of the WPES to determine whether the structural damage occurs or not. This method has been applied in the Runyang Yangtze River Bridge and achieved good results. According to the energy-damage theory, Li et al. [13] proposed a structural damage identification method based on wavelet packet analysis and neural network, which was tested on the Benchmark model and achieved satisfactory identification effects. As a metric of the uncertainty state, information entropy is introduced into the field of structural damage identification. Xie et al. $[14,15]$ constructed structural damage index based on the transfer entropy and approximate entropy of the structural response. By combining information entropy with wavelet analysis technology, Ren et al. [16] proposed to use of the wavelet time entropy and the relative wavelet entropy as structural damage index.

Although the structural damage identification methods have developed significantly, some deficiencies remain. The primary defect is that most existing structural damage index varies with the changes of excitation, therefore, it is difficult to determine that the change of damage index is derived from the changes of excitation or the real damage. In order to solve this problem, a new structural damage identification method based on wavelet packet energy entropy (WPEE) of impulse response is presented. The method firstly extracts the impulse response by natural excitation technique (NExT). Then, a new damage index is constructed based on change rate of WPEE.

The remaining parts of the paper are organized as follows: Section 2 introduces the wavelet packet theory and information theory. Section 3 proposes a new damage index based on change rate of WPEE. Section 4 provides a simulated experiment using a pile structure to verify the effectiveness of the new method, and the conclusion is given in Section 5. 


\section{BACKGROUND}

\subsection{Wavelet Packet Theory}

Wavelet transform is considered as an effective signal processing technology in time-frequency domain. However, wavelet scale function is changed as a binary system, which leads to a poor frequency resolution in the high frequency band. Wavelet packet transform provides a more precise method on signal processing. Further decomposition of high frequency part is carried out by wavelet packet transform. According to the characteristic of the signal, wavelet packet transform can adaptively select the appropriate frequency band to match with the signal spectrum. Therefore, wavelet packet transform can increase the time-frequency resolution.

Wavelet packet is a subdivision of wavelet subspace in accordance with a binary system. In order to achieve wavelet packet, it firstly requires constructing a new space $U_{j}^{n}$ by combining scale subspace $V_{j}$ with wavelet subspace $W_{j}$. Then, it needs to compute the orthonormal basis of the space, the orthonormal basis is the wavelet packet.

If $U_{j}^{0}$ and $U_{j}^{l}$ can be represented as:

$U_{j}^{0}=V_{j}, \quad U_{j}^{1}=W_{j}, \quad j \in Z$

Then, the decomposition relations may be expressed as:

$U_{j+1}^{0}=U_{j}^{0}+U_{j}^{1}, \quad j \in Z$

It is assumed that $\varphi(x)$ and $\psi(x)$ are the orthogonal scale function and wavelet function, respectively. They are defined as follows:

$\left\{\begin{array}{l}\mu_{0}(x)=\varphi(x) \\ \mu_{1}(x)=\psi(x)\end{array}\right.$

A function cluster can be defined in accordance with the following recursive clustering methods:

$u_{2 n}(t)=\sqrt{2} \sum_{k \in Z} h(k) u_{n}(2 t-k)$

$u_{2 n+1}(t)=\sqrt{2} \sum_{k \in Z} g(k) u_{n}(2 t-k)$

where $g(k)=(-1)^{k} h(1-k)$.

When $n=0$, Equation (4) and (5) can be changed as:

$u_{0}(t)=\sum_{k \in Z} h_{k} u_{0}(2 t-k)$

$u_{1}(t)=\sum_{k \in Z} g_{k} u_{0}(2 t-k)$

In the multi-resolution analysis, $\varphi(x)$ and $\psi(x)$ meet the two-scale equations:

$$
\begin{aligned}
& \phi(t)=\sum_{k \in z} h_{k} \phi(2 t-k)\left\{h_{k}\right\}_{k \in z} \in l^{2} \\
& \psi(t)=\sum_{k \in z} g_{k} \psi(2 t-k)\left\{g_{k}\right\}_{k \in z} \in l^{2}
\end{aligned}
$$

$u_{0}(t)$ and $u_{1}(t)$ are the scale function and wavelet function, respectively. The orthogonal wavelet packet can be constructed by $\left\{u_{n}(t)\right\}\left(n \in Z^{+}\right)$.

If $n$ is a parameter of refinement multiplier and $n=2^{l}+m$, then the wavelet packet can be simplified as:

$\psi_{j, k, n}(t)=2^{-j / 2} \psi_{n}\left(2^{-j} t-k\right)$

where $\psi_{n}(t)=2^{-l / 2} u_{n}\left(2^{-l} t\right), \psi_{j, k, n}(t)$ is considered as the wavelet packet with a scale indicator $j$, position indicator $k$ and frequency indicator $n$.

If $g_{j}^{n}(t) \in U_{j}^{n}$, then $g_{j}^{n}$ can be represented as:

$g_{j}^{n}(t)=\sum_{l} d_{l}^{j, n} u_{n}\left(2^{j} t-l\right)$

Wavelet packet decomposition algorithm can be represented as:

$$
\begin{aligned}
& d_{l}^{j, 2 n}=\sum_{k} a_{k-2 l} d_{k}^{j+1, n} \\
& d_{l}^{j, 2 n+1}=\sum_{k} b_{k-2 l} d_{k}^{j+1, n}
\end{aligned}
$$

As the scale increases, the orthogonal wavelet basis functions has a lower frequency resolution, which is the defect of the orthogonal wavelet. Wavelet packet can make further segmentation of frequency band, thereby increasing the frequency resolution.

\subsection{Information Entropy}

Information entropy was proposed by C. Shannon in 1948, which is considered as a measure of the uncertainty of a random event and described by probability distribution function. It is assumed that the probability of each result is the vector $p=\left(p_{1}, p_{2}, \ldots, p_{\mathrm{m}}\right)$, which satisfies the following equation:

$\sum_{i=1}^{m} p_{i}=1$

where $0 \leq p_{i} \leq 1, i=1,2, \cdots m$.

Function $H($.$) was introduced to define the information$ entropy.

$H_{m}(X)=H_{m}\left(\mathrm{p}_{1}, \mathrm{p}_{2}, \cdots, \mathrm{p}_{\mathrm{m}}\right)=-k \sum_{i=1}^{m} p_{i} \log p_{i}$

where $k \geq 0$ is a constant.

$H(m)$ is the information entropy, which represents the uncertainty of a random event by probability distribution function.

\section{A NEW DAMAGE IDENTIFICATION INDEX BASED ON WPEE}

\subsection{Impulse Response Extraction Based on NExT}

NExT is firstly proposed by JAMES and CARNE in 1995 [17], whose basic idea is that the cross-correlation 
function of response signals and impulse response function have a similar form under the white noise excitation. Therefore, the cross-correlation function of response signals can be considered as impulse response to achieve structural damage identification.

The equation of motion for the $n$ DOF dynamic system is represented as:

$$
M \ddot{x}(t)+C \dot{x}(t)+K x(t)=f(t)
$$

where $M$ is the structural mass matrix, $C$ is the structural damping matrix, $K$ is the structural stiffness matrix, $x(t)$ is the displacement vector and $f(t)$ is the input excitation.

When point $k$ is subjected to external excitation $f_{k}(t)$, the measured response of point $i x_{i k}(t)$ can be computed as:

$x_{i k}=\sum_{r=1}^{2 N} \phi_{i r} m_{k r} \int_{-\infty}^{t} e^{\lambda_{r}(t-p)} f_{k}(p) d p$

where $\phi_{i r}$ is the $r$ th mode of point $i, m_{k r}$ is a constant associated with excitation point $k$ and modal order $r$.

If the response of point $j$ is $x_{j k}(t)$, then the crosscorrelation function of $x_{i k}(t)$ and $x_{j k}(t)$ can be computed as:

$$
\begin{gathered}
R_{i j k}=E\left[x_{i k}(t+k) x_{j k}(t)\right]=\sum_{r=1}^{2 N} \sum_{S=1}^{2 N} \phi_{i r} \phi_{j s} m_{k r} m_{k s} \\
\int_{-\infty}^{t} \int_{-\infty}^{t+\tau} e^{\lambda_{r}(t+\tau-p)} e^{\lambda_{r}(t-\tau)} E\left[f_{k}(p) f_{k}(q)\right] d p d q
\end{gathered}
$$

If the excitation point $k$ is the unit impulse force, then the impulse response of point $i$ can be achieved as:

$h_{i k}=\sum_{r=1}^{2 N} \phi_{i r} m_{k r} e^{\lambda_{r} t}$

It is assumed that $f_{k}(t)$ is white Gauss noise, we can get the following equation based on the definition of the crosscorrelation function.

$$
E\left[f_{k}(p) f_{k}(q)\right]=m_{k} \delta(p-q)
$$

where $\delta(t)$ is the impulse function, $m_{k}$ is a constant associated with the excitation point $k$.

Combined Equation (18) with Equation (20), we can get Equation (21).

$$
R_{i j k}(\tau)=\sum_{r=1}^{2 N} \sum_{s=1}^{2 N} \phi_{i r} \phi_{j s} m_{k r} m_{k s} m_{k} \int_{-\infty}^{t} e^{\lambda_{r}(t+\tau-p)} e^{\lambda_{r}(t-\tau)} d p
$$

Integral part of the Equation (21) can be simplified as:

$-\frac{e^{\lambda_{r} \tau}}{\lambda_{r}+\lambda_{s}}=\int_{-\infty}^{t} e^{\lambda_{r}(t+\tau-p)} e^{\lambda_{r}(t-\tau)} d p$

So, Equation (21) can be written as:

$$
R_{i j k}(\tau)=\sum_{r=1}^{2 N} \sum_{s=1}^{2 N} \phi_{i r} \phi_{j s} m_{k r} m_{k s} m_{k}\left(-\frac{e^{\lambda_{r} \tau}}{\lambda_{r}+\lambda_{s}}\right)
$$

If $n_{j r}=\sum_{s=1}^{2 N} \phi_{j s} m_{k r} m_{k s} m_{k}\left(-\frac{1}{\lambda_{r}+\lambda_{s}}\right)$, then the following forms can be obtained:

$$
R_{i j k}(\tau)=\sum_{r=1}^{2 N} n_{j r} \phi_{i r} e^{\lambda_{r} \tau}
$$

Compared Equation (19) with Equation (24), it can be seen that the cross-correlation function of response signals and impulse response can be both expressed as the summation of complex exponential functions. Therefore, the cross-correlation function of response signals can be considered as impulse response to achieve structural damage identification.

\subsection{A New Structural Damage Identification Index}

According to the wavelet theory, when wavelet functions are a set of orthogonal basis functions, the wavelet transform has the property of energy conservation. Therefore, Equation (25) can be provided as:

$\sum_{j=-1}^{-N}\left|x(t), \psi_{j, k}(t)\right|^{2}=\|x\|^{2}$

The definition of wavelet energy is provided as:

$$
E_{j}=\sum_{k}\left|C_{j}(k)\right|^{2}
$$

where $C_{j}(k)$ is the wavelet coefficients of scale $j$.

Wavelet energy represents the quadratic sum of wavelet coefficients under a single scale. So, the total wavelet energy can be expressed as:

$$
E=\sum_{j} E_{j}=\sum_{j} \sum_{k}\left|C_{j}(k)\right|^{2}
$$

The definition of the relative wavelet energy can be provided as:

$p_{j}=\frac{E_{j}}{E}$

$p_{\mathrm{j}}$ can express the energy distribution of different scales. It is a good tool to detect the abnormal points of signals. Based on the information entropy theory and the definition of the relative wavelet energy, the WPEE can be computed as:

$$
S_{w t}=\sum_{j=1} p_{j} \log p_{j}
$$

where $\log$ is the logarithm using base 2 .

In order to accurately describe the changes of the structural state property, the change rate of WPEE is proposed as damage index in this paper, which is defined as follows:

$$
C R(t)=\frac{S_{w t}(t)-S_{w t}(0)}{S_{w t}(0)}
$$


where $S_{w t}(0)$ is WPEE of the intact structure. $S_{w t}(t)$ is WPEE of the structural state at time $t$. $C R$ is the change rate of WPEE.

$C R$ expresses the changes of WPEE under different structure state. Firstly, WPEE of the intact structure is considered as a metric. If the structures don't damage in a period of time, the dynamic characteristic of the structure is relatively stable, the change rate of WPEE is very small. When structural damage occurs, the response signal will change significantly. At this time, WPEE will increase dramatically. Due to this feature, change rate of WPEE can be used to identify the structural damage.

The process of structural damage identification using $C R$ is provided as Fig. (1).

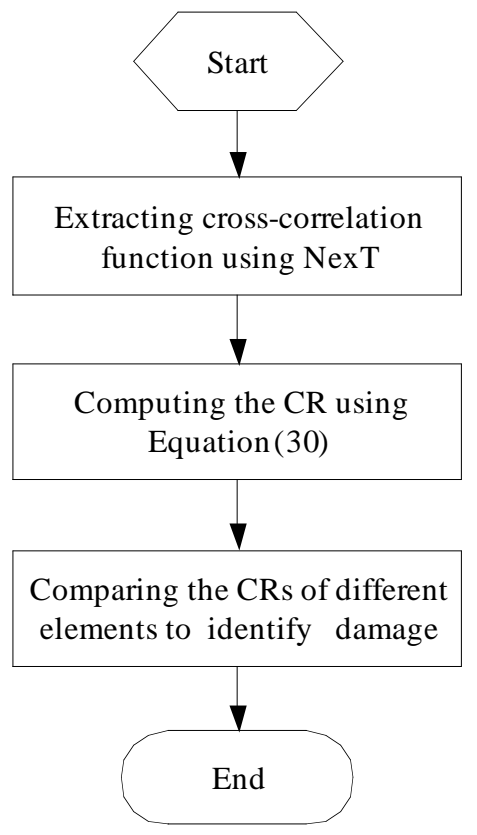

Fig. (1). The flowchart of the proposed method.

\section{DEMONSTRATION CASE}

\subsection{Finite Element Model}

In order to verify the effectiveness of the proposed method, a case study of structural damage identification on a numerically simulated pile structure is provided. The total length of pile is $10 \mathrm{~m}$, the cross-sectional area of the pile is $0.4^{*} 0.4 \mathrm{~m}^{2}$. The finite element (FE) model of the pile is established using commercial software ANSYS, the pile is modeled by the element of Beam 4. The pile is divided into 15 elements, and 16 nodes are generated in total. Material parameters of the pile are set as: the elastic modulus $E=2.5 \times 10^{9} \mathrm{~N} / \mathrm{m}^{2}$, Poisson ratio $\mu=0.17$, density $\rho=2500 \mathrm{~kg} / \mathrm{m}^{2}$. The FE model is shown in Fig. (2).

The white Gauss noise load is generated as input excitation. The duration time of load is set as $5 \mathrm{~s}$, the step is set as 0.01 s. The white Gauss noise load is shown in Fig. (3). Node 1 is set as a reference node, and the response signal of node 1 is shown in Fig. (4).

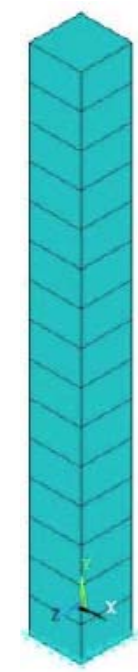

Fig. (2). FE model of the pile structure.

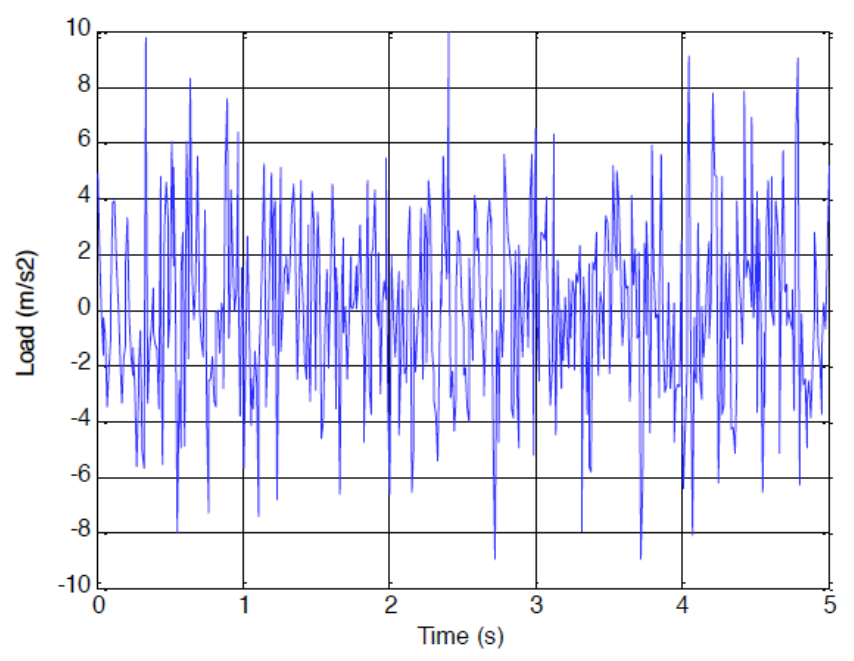

Fig. (3). White Gauss noise excitation.

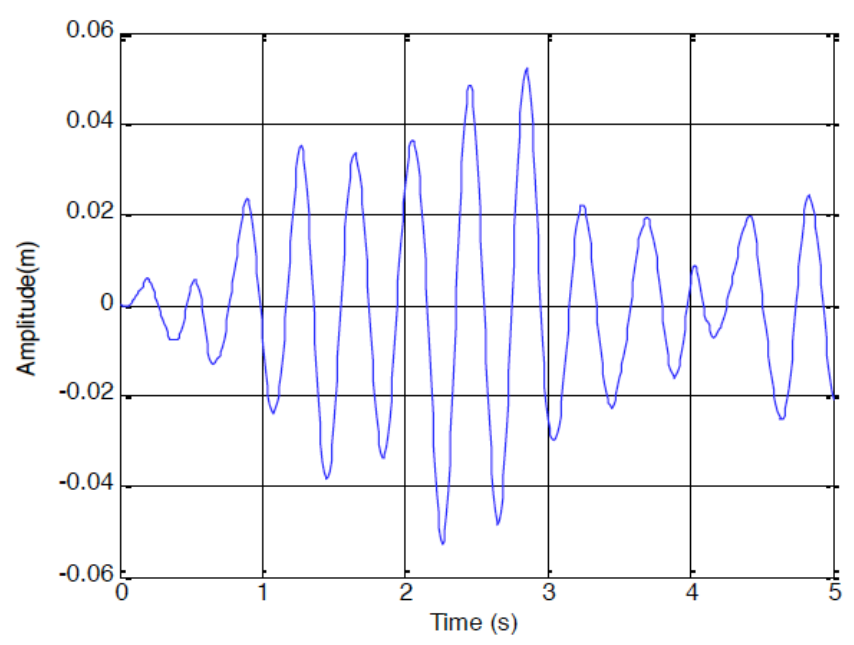

Fig. (4). Response signal of node 1.

\subsection{A Single Damage Identification}

Damage of the element is simulated by reducing its stiffness. In this case study, it is assumed that the damaged 


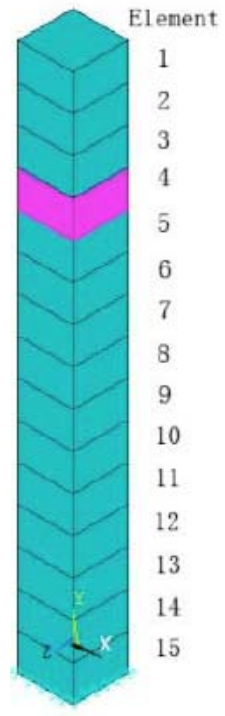

Fig. (5). A single damage location.

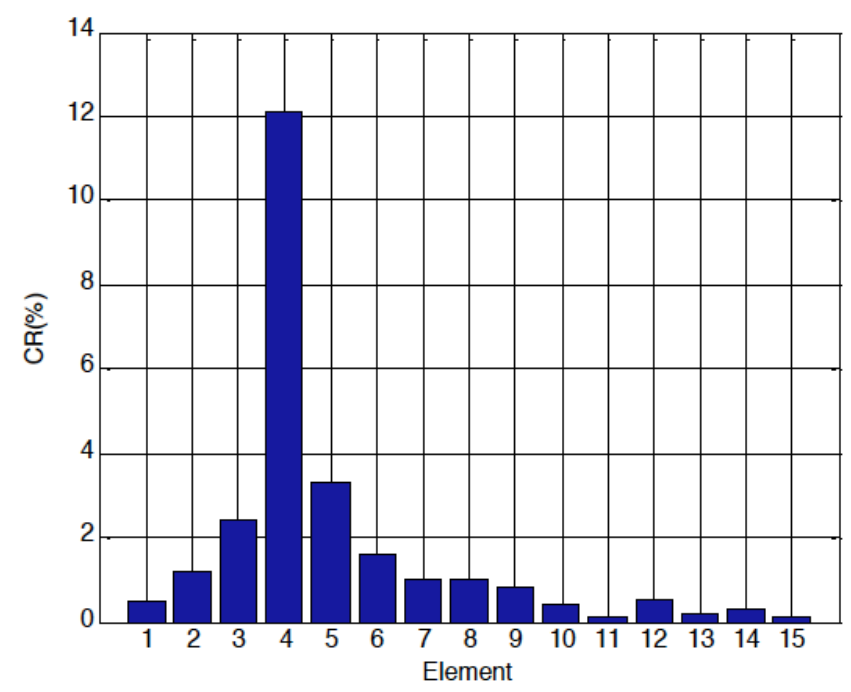

Fig. (6). $C R$ s of all the elements when element 4 is damaged.

element is element 4 , the stiffness of element 4 reduced by $20 \%$. The damage location is shown in Fig. (5).

Node 6 is selected as the response node. The crosscorrelation function of node 1 and node 6 is computed by Equation (24). The CRs of all the elements are computed in accordance with the method in Fig. (1). The result is given in Fig. (6).

In order to prove the validity and superiority of our method, MSECR index proposed in [6] and WPES index proposed in [12] are selected as comparative experiments. The results are shown in Figs. (7 and 8).

\subsection{Multi-Damage Identification}

A new experiment is provided to show the multi-damage identification effect of the proposed method. It is assumed that element 4 and 9 are both damaged (see Fig. 9), the stiffness of element 4 and 9 are reduced by $20 \%$. The experiment result is shown in Fig. (10).

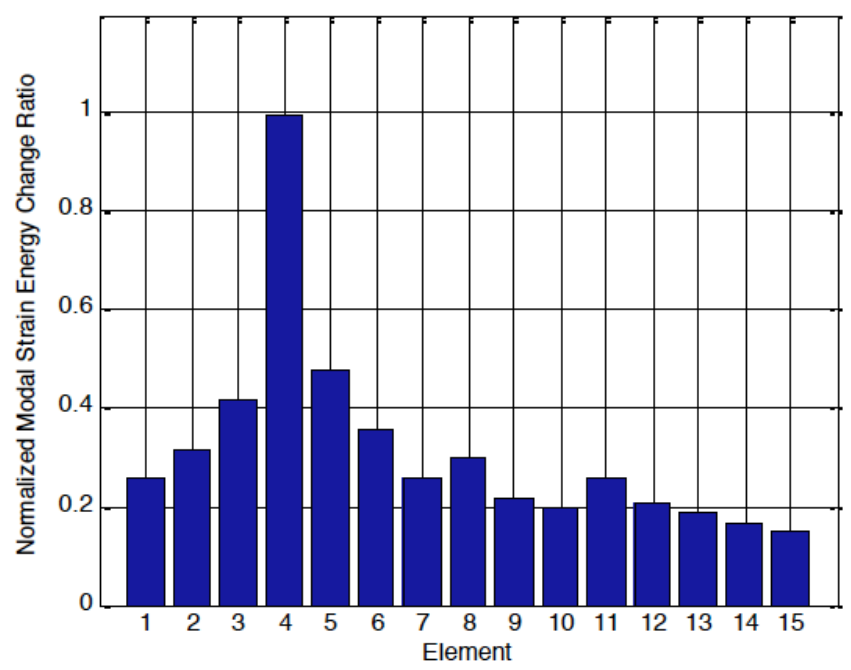

Fig. (7). MSECR index when element 4 is damaged.

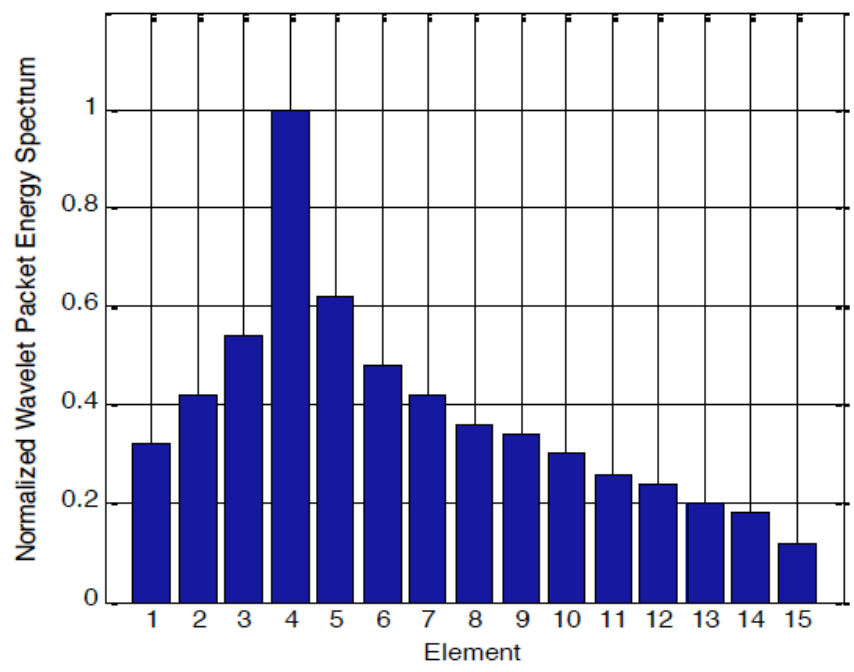

Fig. (8). WPES index when element 4 is damaged.

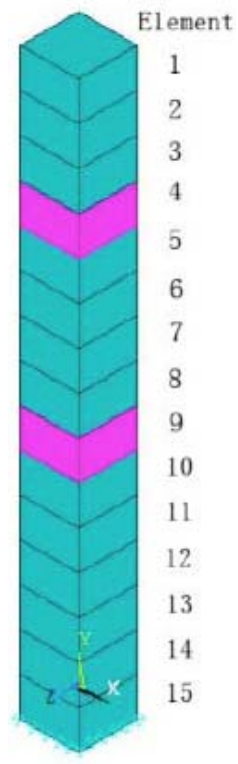

Fig. (9). Multi-damage locations. 


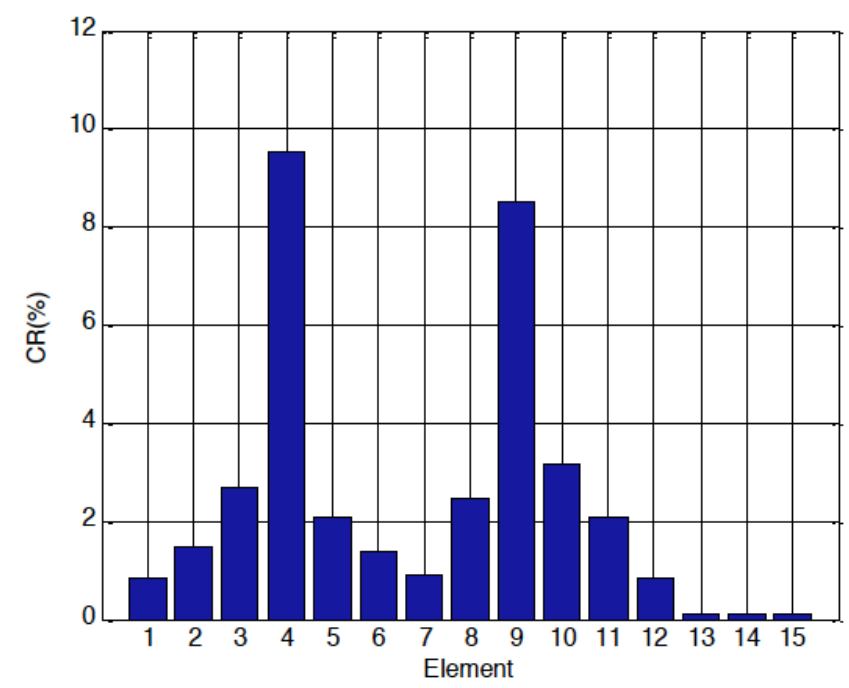

Fig. (10). $C R$ s of all the elements when element 4 and element 9 damages.

MSECR index and WPES index are selected as comparative experiments. The results are shown in Figs. (11 and 12).

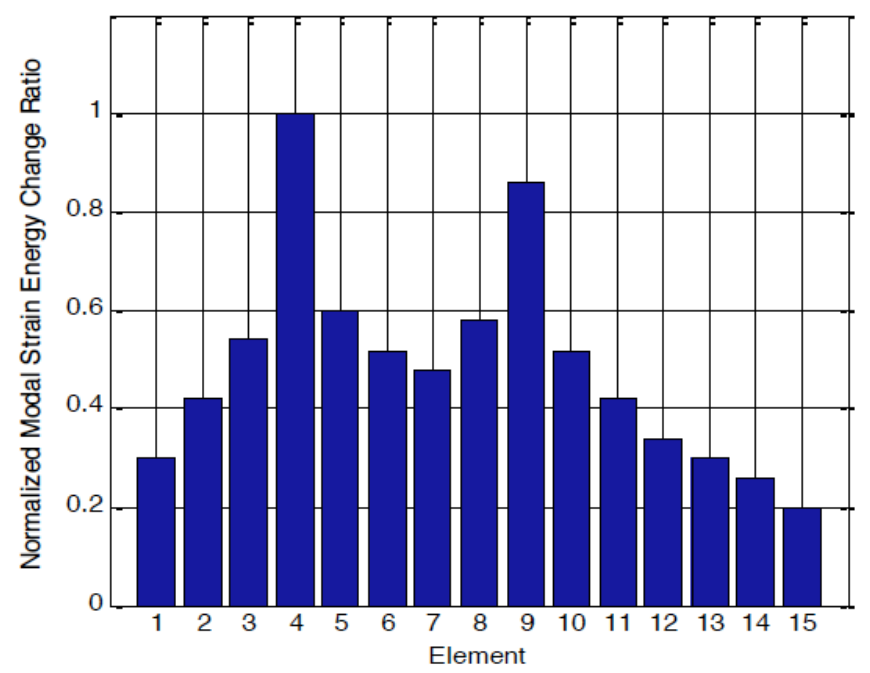

Fig. (11). MSECR index when element 4 and element 9 damages.

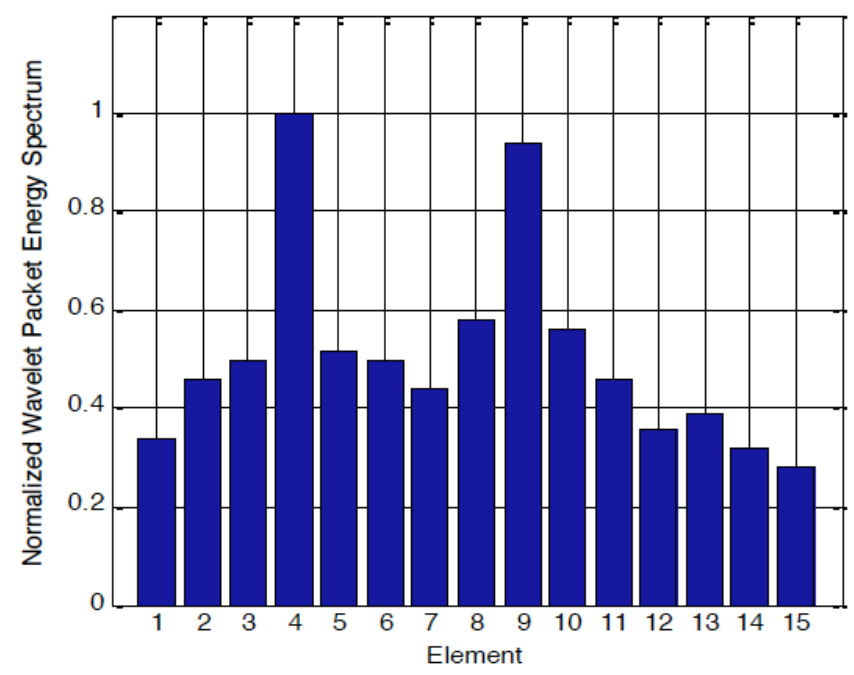

Fig. (12). WPES index when element 4 and element 9 damages.

\subsection{Result Analysis}

As seen in Fig. (6), the $C R$ of element 4 is $12.1 \%$, which is much bigger that the other elements. Therefore, we can obviously identify that the element 4 is damaged. Fig. (9) shows that the $C R$ s of elements 4 and 9 are $9.7 \%$ and $8.4 \%$ respectively, which are both bigger that the other elements. So, the two damages can be both identified by comparing $C R$. As shown in Figs. (7-9), our method can more clearly identify the single damage. From Figs. (10-12), we can see our index is more sensitive for multi-damage identification.

\section{CONCLUSION}

The traditional identification method has a low identification precision. In order to solve this problem, a new structural damage identification method based on WPEE of impulse response is presented. The method firstly achieves the structural impulse response by calculating the crosscorrelation function of the different signals. Then, a new damage index is constructed based on change rate of WPEE. With the case analysis, some conclusions are summarized as follows:

(1) The cross-correlation function of response signals has the similar form with impulse response. Therefore, crosscorrelation function can be considered as impulse response to identify the structural damage.

(2) When structural damage occurs, the WPEE of damaged element will change significantly. By comparing the $C R$ of different elements, the damaged element can be identified accurately. The experiment results show that $C R$ can identify the single damage and multi-damage accurately, which proves $C R$ is an effectiveness structural damage identification index.

\section{CONFLICT OF INTEREST}

The authors confirm that this article content has no conflict of interest.

\section{ACKNOWLEDGEMENTS}

Declared none.

\section{REFERENCES}

[1] J. E. Padgett, and C. Tapia, "Sustainability of natural hazard risk mitigation: Life cycle analysis of environmental indicators for bridge infrastructure," Journal of Infrastructure Systems, vol. 19, no. 4, pp. 395-408, 2013.

[2] D. Balageas, C. P. Fritzen, and A. Güemes, Eds., Structural Health Monitoring, ISTE, London, 2006.

[3] S. F. Jiang, S. Y. Wu, and L. Q. Dong, "A time-domain structural damage detection method based on improved multiparticle swarm coevolution optimization algorithm," Mathematical Problems in Engineering, vol. 2014, Article ID 232763, 2014.

[4] J. Zhang, Y. L. Xu, J. Li, Y. Xia, and J. C. Li, "Statistical momentbased structural damage detection method in time domain," Earthquake Engineering and Engineering Vibration, vol. 12, no. 1, pp. 13-23, 2013.

[5] W. Fan, and P. Qiao, "Vibration-based damage identification methods: a review and comparative study," Structural Health Monitoring, vol. 10, no. 1, pp. 83-111, 2011. 
[6] Z. Y. Shi, S. S. Law, and L. M. Zhang, "Structural damage detection from modal strain energy change," Journal of Engineering Mechanics, vol. 126, no. 12, pp. 1216-1223, 2000.

[7] Y. Liu, M. Y. Fard, and A. Chattopadhyay, "Damage assessment of CFRP composites using a time-frequency approach," Journal of Intelligent Material Systems and Structures, vol. 23, no. 4, pp. 397413, 2012.

[8] A. Trochidis, L. Hadjileontiadis, and K. Zacharias, "Analysis of vibro-acoustic modulations for crack detection: a time-frequency approach based on zhao-atlas-marks distribution," Shock and Vibration, vol. 2014, Article ID 102157, 2014.

[9] H. Kim, and H. Melhem, "Fourier and wavelet analyses for fatigue assessment of concrete beams," Experimental Mechanics, vol. 43, no. 2, pp. 131-140, 2003.

[10] Z. Hou, M. Noori, and S. Amand, "Wavelet-based approach for structural damage detection," Journal of Engineering Mechanics, vol. 126, no. 7, pp. 677-683, 2000.

[11] M. E. Zitto, R. Piotrkowski, A. Gallego, F. Sagasta, and A. B. Climent, "Damage assessed by wavelet scale bands and b-value in dynamical tests of a reinforced concrete slab monitored with acoustic emission," Mechanical Systems and Signal Processing, vol. 60, no. 8, pp. 75-89, 2015.
[12] Y. L. Ding, A. Q. Li, and C. Q. Miao, "Theoretical research on structural damage alarming of long-span bridges using wavelet packet analysis," Journal of Southeast University, vol. 21, no. 4, pp. 459-462, 2005.

[13] T. H. Yi, H. Li, and G. Wang, "Cycle slip detection and correction of GPS carrier phase based on wavelet transform and neural network," In: $6^{\text {th }}$ International Conference on Intelligent Systems Design and Applications: Jinan, 2006, pp. 46-50.

[14] Z. K. Xie, G. H. Liu, and Z. G. Wu, "Dynamic damage identification for beam structures based on transfer entropy," Journal of Zhejiang University, vol. 46, no. 10, pp. 1880-1886, 2012, (in Chinese).

[15] Z. K. Xie, G. H. Liu, and Z. G. Wu, "Damage detection of concrete structure based on approximate entropy," Applied Mechanics and Materials, vol. 226, no. 10, pp. 920-925, 2012.

[16] W. X. Ren, and Z. S. Sun, "Structural damage identification by using wavelet entropy," Engineering Structures, vol. 10, no. 3, pp. 2840-2849, 2008.

[17] G. H. James, T. G. Carne, and J. P. Lauffer, "The natural excitation technique (NExT) for modal parameter extraction from operating structures," International Journal of Analytical and Experimental Modal Analysis, vol. 10, no. 4, pp. 260-277, 1995.

Received: March 17, 2015

Revised: June 02, 2015

Accepted: June 08, 2015

(c) He et al.; Licensee Bentham Open.

This is an open access article licensed under the terms of the Creative Commons Attribution Non-Commercial License (http://creativecommons.org/licenses/ by-nc/3.0/) which permits unrestricted, non-commercial use, distribution and reproduction in any medium, provided the work is properly cited. 